\title{
Interfaces between highly incompatible polymers of different stiffness: Monte Carlo simulations and self-consistent field calculations
}

\author{
M. Müller ${ }^{1,2}$ and A. Werner ${ }^{1}$ \\ ${ }^{1}$ Institut für Physik, Johannes Gutenberg Universität \\ D-55099 Mainz, Germany \\ ${ }^{2}$ Department of Physics, Box 351560, University of Washington, \\ Seattle, Washington 98195-1560
}

June 24, 2021

\begin{abstract}
We investigate interfacial properties between two highly incompatible polymers of different stiffness. The extensive Monte Carlo simulations of the binary polymer melt yield detailed interfacial profiles and the interfacial tension via an analysis of capillary fluctuations. We extract an effective Flory-Huggins parameter from the simulations, which is used in self-consistent field calculations. These take due account of the chain architecture via a partial enumeration of the single chain partition function, using chain conformations obtained by Monte Carlo simulations of the pure phases. The agreement between the simulations and self-consistent field calculations is almost quantitative, however we find deviations from the predictions of the Gaussian chain model for high incompatibilities or large stiffness. The interfacial width at very high incompatibilities is smaller than the prediction of the Gaussian chain model, and decreases upon increasing the statistical segment length of the semi-flexible component.
\end{abstract}

\section{Introduction}

Melt blending of polymers has proven useful in designing new composite materials with improved application properties. In many practical situations the constituents of the blend are characterized by some degree of structural asymmetry. For example, a flexible component might contribute to a higher resistance to 
fracture, while blending it with a stiffer polymer can increase the tensile strength of the material. Since the entropy of mixing in polymeric systems decreases with increasing degree of polymerization, a small unfavorable mismatch in enthalpic interactions, entropic packing effects or the combination of both, generally leads to materials which are not homogeneous on mesoscopic scales, but rather fine dispersions of one component in another. Therefore properties of interfaces between unmixed phases are crucial in controlling the application properties of composites [1] and have found abiding experimental interest [2, 3, 4 , 5.

Recently, the bulk phase behavior and surface properties $[6]$ of polyolefins $[$, 8] with varying microstructure has attracted considerable experimental and theoretical interest. These mixtures are often modeled [7, 9, 10] as blends of polymers with different bending rigidities, the less branched polymer corresponding to the more flexible component. For pure hard core interactions, field theoretical calculations by Fredrickson, Liu and Bates [9], polymer reference interaction site model (P-RISM) computations by Singh and Schweizer[10], lattice cluster theories by Freed and Dudowicz 11 and Monte Carlo simulations 12 find a small positive contribution to the Flory-Huggins parameter $\chi$. Monte Carlo simulations which include a repulsion between unlike species reveal an additional increase of the effective Flory-Huggins parameter with chain stiffness, because a back folding of chains becomes less probable with increasing stiffness and the number of intermolecular contacts increases 12 respectively. Qualitatively similar effects were found analytically in P-RISM 10 and lattice cluster 11 theories.

In spite of their ubiquitous occurrence, interfacial properties in asymmetric blends have attracted comparably little interest. When entropic packing contributions to the Flory-Huggins parameter $\chi$ are small and composition fluctuations are negligible, the self-consistent field theory is expected to yield an adequate quantitative description. Helfand and Sapse 13] extended the selfconsistent field theory to Gaussian chains with different statistical segment lengths. In the limit of infinite long Gaussian chains and strong segregation, they obtained analytical expressions for the interfacial width $w$ and the interfacial tension $\sigma$. Both increase upon increasing the statistical segment length of one component, leaving $\chi$ and the architecture of the other component unaltered.

However, there are other models, that incorporate structural disparities on the monomer level. Freed and coworkers model monomers as clusters of various shape on a lattice 14] and have explored corrections to the energy of mixing and entropic contributions to the Flory-Huggins parameter.

Stiffness disparities have also been investigated using the worm-like chain model[15], which captures the crossover between rod-like behavior on small length scales and Gaussian statistics on length scales much larger than the persistence length. Morse and Fredrickson 16 extended the self-consistent field calculation to a symmetric blend of worm-like chains. For vanishing bending rigidity $\kappa$ they reproduced the Gaussian chain result. In the limit of high bending rigidities and strong segregation $(\kappa \chi \gg 1)$, however, they found that the 
width $w$ of the monomer density profile can be considerably smaller than for a Gaussian chain with the same long distance behavior. At large $\kappa \chi$, increasing the statistical segment length even leads to a decrease of the interfacial width in qualitative contrast to the Gaussian chain result. They also observed that the width of the bond orientation profile is of the order of the persistence length, which is much larger than $w$ in that limit. Thus the interfacial width $w$ and the persistence length constitute two independent length scales of the interfacial profiles. A reduction of the interfacial width in the case of small bending rigidities was obtained numerically by Schmid and Müller 17. They noted that the local structure might become important if its length scale is comparable to the interfacial width; a situation which occurs at rather large incompatibilities.

In the present study we extend our Monte Carlo studies 12] of structural asymmetric blends to the investigation of interfacial properties between well segregated phases of flexible and semi-flexible polymers. We consider rather small bending rigidities of the semi-flexible component, so that the long distance behavior of both species is Gaussian. However, we chose the incompatibility $\chi$ high enough, such that the interfacial width and the persistence length are comparable for the higher bending rigidities. The Monte Carlo simulations highlight the architectural influences and give a detailed picture of interfaces between structural asymmetric polymers. They yield density and orientation profiles for bonds and chains as a whole. Extracting an effective Flory-Huggins parameter $\chi$ from the simulation data, we compare our Monte Carlo results to self-consistent field calculations which take due account of the chain architecture via a partial enumeration procedure 18, 19, 20, and to Gaussian chain results. Therefore we can assess the importance of the level of coarse graining on the interfacial properties.

Our paper is organized as follows: In the next section we describe our polymer model, especially the dependence of single chain properties on the stiffness. We comment on some computational aspects of the Monte Carlo simulations and describe the measurement of the interfacial tension. We also introduce the salient features of our self-consistent field calculations for arbitrary molecular architecture. In the following, we present our simulational results and compare them to the self-consistent field calculations. We close with a brief discussion of our findings and an outlook on future work.

\section{$2 \quad$ Model and technical details}

\subsection{Bond fluctuation model and single chain properties}

In the framework of our coarse grained lattice model, a small number of chemical repeat units, say $3-5$, is mapped onto a lattice monomer, such that the relevant features - chain connectivity and excluded volume interaction between monomeric units - are retained. We use the three dimensional bond fluctua- 
tion model (BFM) 21], which has found widely spread application in computer simulations, because it combines the computational efficiency of lattice models with a rather faithful approximation of continuous space properties. Each effective monomer blocks a cube of 8 neighboring sites from further occupancy on a simple cubic lattice. Due to the extended monomer size, the model captures some nontrivial packing effects. We consider a blend of $n_{A}$ flexible polymers of length $N_{A}$ and $n_{B}$ semi-flexible B-polymers comprising $N_{B}$ monomers in a volume $V$. At a total monomer density $\Phi_{0}=\left(N_{A} n_{A}+N_{B} n_{B}\right) / V=0.5 / 8$, the model reproduces many properties of a dense polymeric melt. We use chain lengths $N=N_{A}=N_{B}=32$ and 64 , which correspond to a degree of polymerization of the order 120 and 240 in more chemically realistic polymer models. Monomers are connected via one of 108 bond vectors with lengths $2, \sqrt{5}, \sqrt{6}, 3$ or $\sqrt{10}$, where here, and henceforth, all lengths are measured in units of the lattice spacing. The large number of bond vectors permits 87 different bond angles.

The persistence length of the semi-flexible B-polymers is tuned by imposing an intermolecular potential, which favors straight bond angles. We use a particular simple choice 12: $E(\theta)=f k_{B} T \cos (\theta)$ where $\theta$ denotes the complementary angle to two successive bonds. Previous Monte Carlo simulations 12 of the bulk thermodynamics for $N=32$ and $f=1.0$ revealed a purely entropic FloryHuggins parameter $\Delta \chi=0.0018(2)$ for the athermal blend. This small value is in good quantitative agreement with theories 9, 10]. These packing effects result in a slight increase of the osmotic pressure with the bending energy, which gives rise to a monomer density difference of about $1 \%$ between the coexisting phases.

Since $\Delta \chi \ll 2 / N=0.0625$ for this combination of chain length and stiffness disparity, we introduce an additional enthalpic repulsion to induce phase separation. For simplicity, these thermal interactions are modeled as a square well potential comprising all 54 neighbor sites up to a distance $\sqrt{6}$. The contact of monomers of the same species lowers the energy by $\epsilon k_{B} T$, whereas the contact of different monomers increases the energy by the same amount. A finite size scaling analysis yields accurate estimates for the critical point of the binary blends $(N=32): \epsilon_{c}=0.01442(6), \phi_{A c}=0.5$ and $\epsilon_{c}=0.0127(1), \phi_{A c}=0.516(10)$ for $f=0$ and $1[12$, respectively. In the present study we chose $\epsilon=0.05$ which corresponds roughly to $\chi \approx 0.27$. This value is much higher than typical values for polyolefin blends [7]. Our results correspond to rather strongly immiscible blends (e.g. interfaces between polystyrene (PS) and polyvinylpropylene PVP [5]).

The conformational data for $N=32$ and $\epsilon=0.05$ as a function of the bending energy $f$ are presented in Fig. 1 and Table 11. The inset shows the growth of the chain extension upon increasing $f$. The ratio between the square end-to-end distance $R^{2}$ and the square radius of gyration $R_{g}^{2}$ remains very close to the Gaussian value 6 (within $5 \%$ even for $f=2$ ). Also the small wave vector 
regime of the single chain structure function

$$
S(q)=\frac{1}{N}\left\langle\left|\sum_{i=1}^{N} \exp \left(i \vec{q}_{i}\right)\right|^{2}\right\rangle
$$

is well describable by a Debye function $S_{D}(q) / N=2\left[\exp \left(-q^{2} R_{g}^{2}\right)-1+q^{2} R_{g}^{2}\right] /\left(q^{2} R_{g}^{2}\right)^{2}$ [22]

for $q<0.3$. Thus the long range behavior of our chains is characterized by Gaussian statistics for all values of the bending energy $f$ studied and we define the statistical segment length $b$ according $b^{2}=R^{2} /(N-1)$. Note that the statistical segment length grows from 3.06 for $f=0$ to 4.63 for $f=2$. This asymmetry in the statistical segment length is of similar magnitude as in polyolefin blends [7].

However, for length scales of the order of the statistical segment length, we find deviations from the Gaussian behavior. The plateau $q^{2} S(q)=12 / b^{2}$ for large $q$ in the Kratky Porod plot is only observed for flexible chains $(f=0)$ and yields a slightly higher estimate for the statistical segment length $b=$ 3.4. For the semi-flexible chains the slope of $q^{2} S(q)$ in the range $0.3<q<1$ increases upon increasing the bending energy. Defining an effective bending rigidity of an equivalent worm-like chain $\kappa=R^{2} / 2\left\langle b^{2}\right\rangle(N-1)\left(\left\langle b^{2}\right\rangle\right.$ : mean squared bond length), $\kappa$ grows from 0.68 to 1.57 ( $\kappa \chi=0.18 \cdots 0.47)$ upon increasing the bending energy $f$. For wave vectors $q R_{g} \approx 2 \pi R_{g} / 2 w$ (denoted by the arrows in the Fig. 1), where $w=3.4$ corresponds roughly to the width of the monomer density profile in the self-consistent field (SCF) calculations, we find deviations from the Gaussian behavior for higher bending energies and anticipate corrections to the predictions of the Gaussian model.

\subsection{Local fluid structure and effective Flory-Huggins pa- rameter $\chi$}

In order to compare our simulational results to self-consistent field (SCF) calculations, which cannot account for the local fluid structure of our model, we have to identify an effective Flory-Huggins parameter $\chi$. For the bulk behavior in the one phase region this has been discussed in ref. [12, 23]: We define a dimensionless monomer density $\phi_{A(B)}$ as the ratio between the local number density of $\mathrm{A}(\mathrm{B})$-monomers and the total monomer density $\Phi_{0}$. Then, the density of intermolecular contacts $n_{A B}$ takes the form:

$\frac{2 n_{A A}}{\Phi_{0} \phi_{A}^{2}}=\Phi_{0} \int_{r \leq \sqrt{6}} d^{3} r g_{A A}(r) \equiv z_{A A} \quad$ and $\quad \frac{n_{A B}}{\Phi_{0} \phi_{A} \phi_{B}}=\Phi_{0} \int_{r \leq \sqrt{6}} d^{3} r g_{A B}(r) \equiv z_{A B}$

where $g_{I J}$ denotes the $I J$ interchain correlation function, which is normalized such that $g_{I J}(r \rightarrow \infty)=1$. The integration is extended over the spatial extension of the square well potential and $z_{I J}$ corresponds to the effective coordination number of the Flory-Huggins lattice. If the coupling between chain conformations and effective monomer repulsion is negligible, only the intermolecular 
energy drives the phase separation. In this case (as we shall see in the next subsection), the $\chi$ parameter takes the form: $\chi=\epsilon\left(z_{A A}+2 z_{A B}+z_{B B}\right) / 2 k_{B} T$, where $z_{I J}$ denote the coordination numbers obtained from the intermolecular pair-correlation functions. At the critical temperatures the coordination numbers have been measured in the simulations at composition $\phi_{A}=\phi_{B}=1 / 2[12]$. From its very definition the Flory-Huggins parameter $\chi$ accurately describes the intermolecular interaction energy, and it agrees nicely with values obtained from the semi-grandcanonical equation of state and the estimate from the long wavelength behavior of the collective structure factor. It also yields estimates of the critical temperature, which agree with the Monte Carlo results up to $1 / \sqrt{N}$ corrections due to composition fluctuations 23.

In the pure system, the intermolecular coordination number of the flexible component is lower than the corresponding value for the semi-flexible chains 12. The number of intramolecular contacts 24 is higher for the flexible chains. Therefore, the $\chi$-parameter grows upon increasing $f[12$. Due to the larger chain extension for the semi-flexible component, the correlation hole has a larger spatial extent, but is more shallow. The intermolecular pair correlation function is presented in the inset of Fig. 2. Due to the extended monomer size $g(r)$ vanishes for distances $r<2$. At short distances, the presence of single site vacancies introduces local packing effects, which gives rise to several neighbor shells in the fluid. The extended structure of the polymer manifests itself in a reduction of contacts with other chains on the length scale of the end-to-end distance. On short distances, the intermolecular pair-correlation function for the stiffer chains is larger than for the flexible ones. For flexible chains it is possible to separate the monomeric packing effect from the polymeric correlation hole by dividing $g(r)$ by its monomeric equivalent 23], which exhibits only packing effects. The ratio $g(r) / g_{N=1}(r)$ presents the conditional probability of finding a monomer of a different chain at a distance $r$, if there would be one in the monomer system. This ratio, presented in Fig. 2, is a rather smooth function, indicating, that the chain connectivity hardly affects the monomeric packing. If the correlation hole would be characterized by a single length scale, i.e. the end-to-end distance $R$ in the Gaussian chain model, one expects a scaling behavior of the form:

$$
1-\frac{g(r)}{g_{N=1}(r)}=\frac{N}{R^{3}} f\left(\frac{r}{R}\right)
$$

Such a scaling plot is shown in Fig. 2. The data collapse well for the different bending rigidities at large distances, whereas there are deviations for small distances. This is a further indication, that the chain structure is characterized by two independent length scales, the end-to-end distance and the persistence length.

In the well segregated regime (far below the critical temperature), it is very difficult to measure the $A B$ intermolecular correlation function in the bulk. Therefore, unlike ref. [12], we make an additional ad-hoc assumption: $z_{A B}=\left(z_{A A}+z_{B B}\right) / 2$. For symmetric blends near the critical point P-RISM 
calculations 25 predict that deviations from this behavior die out with growing chain length like $1 / \sqrt{N}$. However, the validity of this random-packing like assumption for highly incompatible structural asymmetric blends is not obvious.

We explore the interfacial structure by simulating a system in a $L \times L \times$ $2 L$ geometry with $L=64$ and periodic boundary conditions in the canonical ensemble. The system contains two interfaces parallel to the $x y$ plane. The chain conformations are generated via local monomer displacements and slithering snake moves, which are applied at a ratio 1:3 (except for $f=0$, where only local monomer displacements were employed). The systems were equilibrated over 125,000 attempted local moves per monomer (AMM) and 375,000 slithering snake tries per chain (SS). Every 12,500 AMM and 37,500 SS movements a configuration was stored for detailed analysis, at least 898 configuration were generated. We use a trivial parallelization strategy on a CRAY T3E, running typically 8 or 32 configurations in parallel.

Profiles across the interface are measured according to the following procedures: "Apparent" profiles are obtained by locating the instantaneous position of the interface across the whole lateral system extension in each snapshot and averaging over profiles with respect to the instantaneous, but laterally averaged midpoint. These profiles exhibit a system size dependent broadening due to capillary fluctuations, which is not accounted for in the SCF calculations. To avoid this broadening, we define "reduced" profiles by laterally dividing the system into subsystems of size $B \times B$. We choose $B=16$. One could reduce the effect of capillary fluctuations further by chosing a smaller block size $B$, however, one should take care not to cut off "intrinsic" fluctuations 26]. Since on the scale $B$ fluctuations are still reasonably described by a Helfrich Hamiltonian 27] (see below), our block size $B$ is larger than the length scale of "intrinsic" fluctuations. This is consistent with Semenov's 26] estimate for the corresponding length scale $L_{\text {cutoff }}=\pi w \approx 10<B$. Thus this averaging procedure reduces the influence of capillary fluctuations, but does not eliminate it completely 28 .

The presence of an interface gives rise to a spatial dependence of the local monomer densities and chain conformations, which in turn is reflected in the intermolecular pair correlation functions. In Fig. 3 we present the reduced profiles of the intermolecular and intramolecular coordination numbers as a function of the distance from the center of the interface for the bending energies $f=0$ and 2 . The individual coordination numbers exhibit a considerable spatial dependence; this is however partially due to the spatial range of interactions and the remaining capillary fluctuations 29. To illustrate the effect we plot the apparent and reduced profile of the $\mathrm{AB}$ intermolecular coordination number. The value at the center of the interface increases upon reducing $B$; the intrinsic value can not be estimated from these data with high precision. However, the average value of the "reduced" profile is close to $z_{A B}=\left(z_{A A}+z_{B B}\right) / 2$, the value used in the SCF calculations.

The total number of intermolecular contacts $z^{\text {inter }}=\left(n_{A A}^{\text {inter }}+n_{B B}^{\text {inter }}+\right.$ $\left.n_{A B}^{\text {inter }}\right) / \Phi_{0}\left(\phi_{A}+\phi_{B}\right)^{2}$ is much less sensitive to the intrinsic (local) profiles and 
shows a gradual transition between the corresponding bulk values, with a reduction at the center of the interface [30] of about $8 \%$. This spatial dependence of the effective Flory Huggins parameter is neglected. Interestingly, the sum of all contacts $z^{\text {all }}$ (both intermolecular and intramolecular) is largely independent of the stiffness or the distance from the interface, i.e. the bending energy or the unfavorable interactions at the interface causes the chains to rearrange (e.g. exchange unfavorable interchain contacts by energetic favorable intrachain contacts) but hardly affect the structure of the underlying monomer fluid.

Therefore, the local fluid structure is dominated by the packing constraints and the excluded volume interactions. The chain connectivity, bending energies, and the thermal interactions are of minor importance for the monomer fluid. The chain conformations are strongly influenced by the bending energies but depend only slightly on the thermal interactions. The Flory-Huggins parameter is determined by the thermal interactions and also depends on the bending energies via the correlation hole effect. The disparity in the packing behavior of the flexible and the stiff polymers is of minor importance for $\chi$ for the chain lengths studied.

\subsection{Measuring the surface tension via the capillary fluc- tuation spectrum}

Due to the stiffness disparity between the species, straightforward application of semi-grandcanonical identity changes between different polymer types are rather inefficient (note that the efficiency drops by about 3 orders of magnitude 12 upon increasing $f$ from 0 to 1 for $N=32$ ) and therefore limited to small chain length and stiffness. Measurement of the interfacial tension via the reweighting of the composition distribution, which has been successfully applied to structural symmetric blends $(f=0)$, is therefore difficult. In principle, the interfacial tension can be determined via the anisotropy of the pressure tensor. This method has been successfully applied in off-lattice simulations [31, but the generalization to lattice models is difficult]32]. However, the spectrum of capillary fluctuations offers an alternative 33 for measuring the interfacial tension; a method which does not rely on identity switches. Let $u(x, y)$ be the local interfacial position. Then the free energy cost for deviations from a flat planar interface is given by the Helfrich expression 27:

$$
\mathcal{H}=\int d x d y \frac{\sigma}{2}(\nabla u)^{2}+\cdots
$$

where higher order gradient terms are neglected. In our simulation, we define local $x$ - and $y$-averaged interface positions by minimizing the quantity

$$
\left|\sum_{z=u(y)-6}^{u(y)+6} \sum_{x=0}^{x=L-1}\left(\phi_{A}(x, y, z)-\phi_{B}(x, y, z)\right)\right|
$$


for the $x$-averaged position $u(y)$ and a similar expression for the $y$-averaged one. This averaged interfacial position is Fourier decomposed according to: $u(y)=\frac{a_{0}}{2}+\sum_{l=0}^{L / 2} a\left(q_{l}\right) \cos \left(q_{l} y\right)+b\left(q_{l}\right) \sin \left(q_{l} y\right)$ with $q_{l}=2 \pi l / L$. The Helfrich Hamiltonian predicts that the Fourier components $a\left(q_{l}\right)$ and $b\left(q_{l}\right)$ are Gaussian distributed with a width

$$
\frac{2}{L^{2}\left\langle a^{2}(q)\right\rangle}=\frac{2}{L^{2}\left\langle b^{2}(q)\right\rangle}=\frac{\sigma}{k_{B} T} q^{2}
$$

In Fig. 4 we present the distribution of the Fourier components for two different bending energies $f=0,2$ and the 4 smallest wave vectors $q$. This long wavelength part of the fluctuation spectrum is well described by the quadratic Helfrich expression. The straight line marks the expected Gaussian distribution for the Fourier amplitudes, to which the simulation data comply. The inverse width of the distribution determines the interfacial tension. The extracted value for the symmetric blend agrees with the independent measurement obtained via the reweighting scheme 30. (The latter scheme measures the interfacial free energy via the ratio of the probability for finding the system in a homogeneous bulk state or a configuration comprising two interfaces. ) To estimate the errors of measuring the interfacial tension via the capillary fluctuation spectrum, it would be desirable to increase the lateral system size. However, the error in extrapolating the simulation data to $q \rightarrow 0$ is smaller than $7 \%$. Thus the analysis of the capillary fluctuation spectrum is an efficient alternative for measuring interfacial tensions in structurally asymmetric systems; the results are compared to the predictions of the SCF calculations in Sec. IIIa.

\subsection{Self-consistent field calculations}

The mean field approach is similar to Helfand 34, 13], Noolandi [35], and Shull[36], except for the treatment of the chain architecture 19. The partition function of a binary polymer blend has the general form 37]:

$$
\mathcal{Z} \sim \frac{1}{n_{A} ! n_{B} !} \int \Pi_{\alpha=1}^{n_{A}} \mathcal{D}\left[r_{\alpha}\right] \mathcal{P}_{A}\left[r_{\alpha}\right] \Pi_{\beta=1}^{n_{B}} \mathcal{D}\left[r_{\beta}\right] \mathcal{P}_{B}\left[r_{\beta}\right] \exp \left(-\frac{\Phi_{0}}{k_{B} T} \int d^{3} r \mathcal{E}\left(\hat{\phi}_{A}, \hat{\phi}_{B}\right)\right)
$$

where the functional integrals $\mathcal{D}[r]$ sum over all polymer conformations and $\mathcal{P}[r]$ denotes the probability distribution characterizing the noninteracting, single chain conformations. $\mathcal{E}$ represents a segmental interaction free energy, and the dimensionless monomer density takes the form 337:

$$
\hat{\phi}_{A}(r)=\frac{1}{\Phi_{0}} \sum_{\alpha=1}^{n_{A}} \sum_{i_{A}=1}^{N_{A}} \delta\left(r-r_{\alpha, i_{A}}\right)
$$

where the sum runs over all monomers in the A-polymer $\alpha$. A similar expression holds for $\hat{\phi}_{B}(r)$. 
The segment free energy $\mathcal{E}$ comprises two contributions: a free volume part arising from hard core interactions and an energetic term from the thermal interactions. Since the melt is nearly incompressible, we approximate the free volume part by a simple quadratic expression introduced by Helfand [34], which reproduces the relative reduction of the total monomer density by about $4 \%$ [17]. However the difference of the bulk densities of the coexisting phases has a different sign in the simulations and than in the SCF calculations. In the simulations the higher osmotic pressure of the semi-flexible component results in a slightly lower bulk density of the semi-flexible component in the simulations, an effect neglected in the SCF calculations. Moreover, in the SCF calculations the more negative intermolecular energy density (see below) of the B component results in a slightly higher density of semi-flexible polymers. The total density differences between the coexisting phases is however only about $1 \%$. The pairwise intermolecular interactions $V_{I J}(r)(I, J=\mathrm{A}, \mathrm{B})$ are treated as point interactions of strength $\epsilon z_{I J} \delta(r) / \Phi_{0} . z_{I J}$ parameterizes the local fluid structure of the underlying microscopic model, as discussed above. The coupling between individual chain conformations and the coordination numbers, which results in the spatial dependence of the $\chi$-parameter observed in the simulations, is neglected. Furthermore, we ignore purely entropic contributions (which have been determined to be small by Monte Carlo simulations) and do not include orientation dependent segmental interactions, which will eventually lead to a nematic phase at much higher bending energies $f$. Thus we take the interactions to be

$$
\frac{\mathcal{E}\left(\phi_{A}, \phi_{B}\right)}{k_{B} T}=\frac{\zeta}{2}\left(\phi_{A}+\phi_{B}-1\right)^{2}-\frac{\epsilon z_{A A}}{2} \phi_{A}^{2}-\frac{\epsilon z_{B B}}{2} \phi_{B}^{2}+\epsilon z_{A B} \phi_{A} \phi_{B}
$$

The inverse compressibility $\zeta$ has been measured in simulations of the athermal model; $\zeta=4.1$ [38]. A Hubbard-Stratonovich transformation rewrites the many chain problem in terms of independent chains in external, fluctuating fields $W_{A}$ and $W_{B}$.

$$
\mathcal{Z} \sim \int \mathcal{D}\left[W_{A}, W_{B}, \Phi_{A}, \Phi_{B}\right] \exp \left(-\mathcal{F}\left[W_{A}, W_{B}, \Phi_{A}, \Phi_{B}\right] / k_{B} T\right)
$$

where the free energy functional is defined by

$$
\begin{aligned}
\frac{\mathcal{F}\left[W_{A}, W_{B}, \Phi_{A}, \Phi_{B}\right]}{\Phi_{0} k_{B} T V}= & \frac{\bar{\phi}_{A}}{N_{A}} \ln \bar{\phi}_{A}+\frac{\bar{\phi}_{B}}{N_{B}} \ln \bar{\phi}_{B}+\frac{1}{V} \int d^{3} r \mathcal{E}\left(\Phi_{A}, \Phi_{B}\right) \\
& -\frac{1}{V} \int d^{3} r\left\{W_{A} \Phi_{A}+W_{B} \Phi_{B}\right\}-\frac{\bar{\phi}_{A}}{N_{A}} \ln q_{A}\left[W_{A}\right]-\frac{\bar{\phi}_{B}}{N_{B}} \ln q_{B}[\text { [WH }] \text { ] }
\end{aligned}
$$

$\bar{\phi}_{A}=\frac{n_{A} N_{A}}{\Phi_{0} V}=1-\bar{\phi}_{B}$ denotes the average A-monomer density and $q_{A}\left[W_{A}\right]$ the single chain partition function in the external field $W_{A}$

$$
q_{A}\left[W_{A}\right]=\frac{1}{V} \int \mathcal{D}_{1}[r] \mathcal{P}_{A}[r] \exp \left(-\Phi_{0} \int d^{3} r \hat{\phi}_{A} W_{A}\right)
$$


respectively. The leading contributions to the partition function stem from those values $\phi_{A}, \phi_{B}, w_{A}, w_{B}$ of the collective variables which extremize the free energy functional, and the mean field approximation amounts to retaining only these contributions. The values are determined by:

$$
\begin{aligned}
\frac{\delta \mathcal{F}}{\delta \phi_{A}} & =0 \quad \Rightarrow \quad w_{a}=\frac{\delta}{\delta \phi_{A}} \int d^{3} r \mathcal{E}\left(\phi_{A}, \phi_{B}\right)=\zeta\left(\phi_{A}+\phi_{B}-1\right)-\epsilon z_{A A} \phi_{A}+\epsilon z_{A}\left(\phi_{3}\right) \\
\frac{\delta \mathcal{F}}{\delta w_{A}} & =0 \Rightarrow \phi_{A}=\frac{\bar{\phi}_{A} V}{N_{A} q_{A}} \frac{\delta q_{A}}{\delta w_{A}}
\end{aligned}
$$

and similar expressions for $w_{B}$ and $\phi_{B}$. The saddle point integration approximates the original problem of mutually interacting chains by one of a single chain in an external field, which is determined, in turn, by the monomer density. Composition fluctuations are ignored, but the coupling between chain conformations (e.g. orientations) and the monomer density is retained. The free energy of a homogeneous system takes the Flory-Huggins form:

$\frac{\mathcal{F}}{\Phi_{0} k_{B} T V}=\frac{\bar{\phi}_{A}}{N_{A}} \ln \left(\bar{\phi}_{A}\right)+\frac{1-\bar{\phi}_{A}}{N_{B}} \ln \left(1-\bar{\phi}_{A}\right)-\frac{1}{2} \epsilon\left\{\left(z_{A A}+2 z_{A B}+z_{B B}\right) \bar{\phi}_{A}^{2}-2\left(z_{A B}+z_{B B}\right) \bar{\phi}_{A}+z_{B B}\right\}$

where we identify the Flory-Huggins parameter $\chi=\left(z_{A A}+2 z_{A B}+z_{B B}\right) \epsilon / 2$. In the strongly segregated regime, the free energy of a system containing one interface is given by: $\mathcal{F}=-\epsilon\left(\bar{\phi}_{A} z_{A A}+\left(1-\bar{\phi}_{A}\right) z_{B B}\right) \Phi_{0} k_{B} T V / 2+\sigma k_{B} T L^{2}$. The definition of the interfacial tension as the difference of the free energy of a system containing an interface and the homogeneous bulk system corresponds literally to the measurement of the interfacial tension via the reweighting scheme [30] in the Monte Carlo simulations. As shown in Sec. IIc for the symmetric blend, these values agree with the measurement of the interfacial tension via the capillary fluctuation spectrum, so that we can compare the results of the SCF calculations and the values extracted from the capillary wave spectrum quantitatively.

For the special cases of Gaussian chains 34, 39] and worm-like polymers 15 , 16. one can treat the single chain problem in an arbitrary external field in limiting cases (e.g $N \rightarrow \infty$ ) analytically. For general parameters, however, one has to resort to numerical procedures even for these simple models. The BFM chains used in the simulations are characterized by structure on different length scales. The conformations are rod-like for length smaller than the persistence length, which depends on the bending energy $f$. On intermediate length scales, they obey self-avoiding walk statistics, while on the largest scale, the excluded volume interactions are screened in the melt, and the chains exhibit Gaussian statistics. Since we want to explore dependence on the explicit chain structure, we evaluate the single chain partition function via a partial enumeration scheme, introduced by Szleifer and coworkers [18. The method is conceptually straightforward and applicable to arbitrary architecture 19, 20. It can use experimental or simulational data as input. Note that no adjustable parameters are involved 
in the chain structure (such as the statistical segment length in the Gaussian model or the bond length and the bending rigidity in the worm-like polymer model) and the chain structure is correctly represented on all length scales. Using Monte Carlo simulations of the pure melt, we generated 40,960 independent polymer conformations for each bending energy. Rotating and translating those original conformations, we obtain a sample of 7,864,320 polymer conformations for chain length $N=32$. (Note only the $z$ coordinates of the chains are employed for a flat interface parallel to the $x y$ plane.) For $N=64$ we use twice as many conformations. Within this framework, the A-monomer density (c.f. eq. 14) is simply the statistical average of independent A-polymers in the external field $w_{A}$ :

$$
\phi_{A}=\bar{\phi}_{A} \frac{\sum_{\alpha=1}^{C} \frac{1}{N_{A}} \sum_{i=1}^{N_{A}} V \delta\left(r-r_{\alpha, i}\right) \exp \left(-\sum_{i=1}^{N_{A}} w_{A}\left(r_{\alpha, i}\right)\right)}{\sum_{\alpha=1}^{C} \exp \left(-\sum_{i=1}^{N_{A}} w_{A}\left(r_{\alpha, i}\right)\right)}
$$

Other single chain quantities are given by corresponding averages over independent chains in the fields $w_{A}$ and $w_{B}$.

The set of nonlinear equations is expanded in a Fourier series 40 and solved by a Newton-Raphson like method. Convergence is usually reached within 3-6 steps. The evaluation of the partition function [20] in the external fields poses rather high memory demands (several Gbytes). Therefore we employ a CRAY T3E, assigning a subset of conformations to each processing element. Typically we use 64 or 128 processors in parallel, and the program scales very well with the number of processors employed 20]. One needs about 1200 seconds for each set of parameters, which is roughly 2 orders of magnitude less than for the detailed Monte Carlo simulations.

\section{Comparison between Monte Carlo simula- tions and self-consistent field (SCF) calcula- tions}

In the following we compare our Monte Carlo simulations to the results of the SCF calculations. Both, large length scale thermodynamic properties (e.g. interfacial tension) as well as the local interfacial structure (e.g. orientation of individual bonds) are investigated. The temperature dependence of most quantities for symmetric blends $(f=0)$ has been studied previously 30 and compared to predictions of the Gaussian and worm-like chain model[17]. The results for vanishing bending energy compare well to our calculations. 


\subsection{Interfacial tension}

The interfacial tension $\sigma$ between the coexisting phases has an important impact on the morphology of the compound material 41. The control of domain size and shape is a key to tailoring the application properties of the blend. The size of minority droplets often is the smaller, the smaller the interfacial tension between the coexisting phases 41, 42. In the strong segregation limit, Helfand and Sapse 13 obtained for infinite long Gaussian chains in an incompressible blend the analytic expression:

$$
\sigma=\Phi_{0} \sqrt{\chi / 6}\left(\frac{2}{3} \frac{b_{A}^{2}+b_{A} b_{B}+b_{B}^{2}}{b_{A}+b_{B}}\right)
$$

The interfacial tension $\sigma$ grows upon increasing the bending energy (i.e. the statistical segment length) of the semi-flexible component. This behavior is presented in Fig. 5, as well as our simulation results and the SCF calculations, which take account of the detailed chain architecture. All data exhibit an increase of the interfacial tension of about $30 \%$. The simulation data and the SCF calculations agree nicely on the growth of the interfacial tension upon increasing the bending energy. The almost quantitative agreement indicates that our identification of the $\chi$-parameter yields reasonable results for structural asymmetric mixtures.

However, the Gaussian chain result is about a factor 1.3 higher than the simulation data. Recently, Ermoshkin and Semenov 43. calculated corrections to the interfacial tension due to effects of finite chain length $N$. For symmetric blends, they found that chain end effects reduce the interfacial tension by a factor $(1-4 \ln 2 / \chi N) \approx 0.67$, which accounts well for the discrepancies between the Helfand-Sapse result and the Monte Carlo data. Similar reductions are found in numerical SCF calculations 17, 36].

Note that purely entropic, packing contributions to the Flory-Huggins parameter $\Delta \chi$ are less than $1 \%$ of the total $\chi$ value for $f=1$. That is somewhat smaller than the uncertainties in identifying the enthalpic contributions of $\chi$ and the accuracy of our interfacial tension measurement in the simulations. Therefore, purely entropic effects derived from packing are irrelevant to the interfacial behavior for the chain lengths, stiffness asymmetries, and temperatures investigated in the present study.

\subsection{Monomer density profiles}

Another important characterization of the interface are the density profiles of the individual components. Experiments 44] indicate, that entanglements in

the interfacial zone are of major importance for the mechanical properties of the blend. Of course, our chain lengths are too small to observe entanglements, however static properties can be extracted from our simulation data. The density profiles obtained from the SCF calculation are presented in Fig. 6, as well 
as the "apparent" profiles in the Monte Carlo simulation. The width of the apparent profile in the Monte Carlo simulations is about a factor 1.5 larger than the SCF result; this is not unexpected, because capillary fluctuations increase the squared width by a term proportional to $\ln (L) / \sigma$. However, the profiles are qualitative similar: both data show a reduction of the total monomer density at the center of the interface (the relative reduction is roughly $\chi / 2 \zeta[30]$ ) and almost no dependence on the bending energy $f$.

The dependence of the interfacial width on the bending energy is shown in Fig. 7 [45]. The width $w_{r}$ of the reduced profile is smaller than the apparent width $w_{a}$, and agrees better with the SCF results. Due to remaining capillary wave effects it is an upper bound on the intrinsic width. All profiles presented below are obtained by the reduced averaging procedure. The excess energy density of the interface can also be used to estimate the intrinsic width. Since the relative increase in interfacial area due to capillary fluctuations is of the order $\sigma \ln L / L^{2}$, this quantity (as well as the interfacial tension) is not strongly affected by fluctuations of the local interfacial position. A tanh-shaped profile $\phi_{A}=\frac{1}{2}\left(1+\tanh \frac{z}{w}\right)$ yields in the SCF framework:

$\frac{e_{s}}{k_{B} T}=\Phi_{0} \int d z\left\{-\frac{\epsilon z_{A A}}{2} \phi_{A}^{2}-\frac{\epsilon z_{B B}}{2} \phi_{B}^{2}+\epsilon z_{A B} \phi_{A} \phi_{B}\right\}-\frac{\Phi_{0} L\left(z_{A A}+z_{B B}\right)}{4} \approx \frac{1}{2} w_{e} \Phi_{0} \chi$

where we have assumed incompressibility and neglected the finite range of interactions and any contribution of the intramolecular interactions to the excess energy density. Of course, this measure relies crucially on the identification of the Flory-Huggins parameter. However, the method is computational very convenient and can be combined 33] with the reweighting methods of measuring interfacial tensions. It results in values which are between the reduced width and the SCF results, which shows again the consistent parameterization of the local fluid structure. The Gaussian chain model for $N \rightarrow \infty$ predicts for symmetric blends $(f=0)$ a width which is about $20 \%$ smaller than the SCF result. SCF calculations 17 of Gaussian chains with the same long distance behavior and which include chain end effects and the finite compressibility agree within $2 \%$ with our results for symmetric, flexible mixtures. An increase of the chain length from $N=32$ to 64 reduces the effective $\chi$-parameter by $4 \%$ and reduces the broadening due to finite chain length effects. The latter effect is stronger, such that the width decreases slightly.

Most notably, the apparent width of the Monte Carlo data, the energetic width $w_{e}$ and the results of the SCF calculations show almost no dependence on the bending energy $f$, whereas the analytic expression obtained by Helfand and Sapse

$$
w=\sqrt{\frac{b_{A}^{2}+b_{B}^{2}}{12 \chi}}
$$

predicts an increase of about $28 \%$ due to the variation of $b_{B}$. Taking account 
of the stiffness dependence of the effective Flory-Huggins parameter $\chi$, the formula above predicts an increase of $21 \%$. Qualitatively, calculations for worm-like chains [16, 17] indicate that increasing the bending rigidity results in a reduction of the interfacial width compared to the Gaussian chain result. For the present combination of parameters, both effects seem to cancel, resulting in an interfacial width, which is nearly independent of the bending rigidity. For lower incompatibilities, the interfacial width is larger, the Gaussian description on the length scale of the interfacial width becomes more appropriate. Therefore the difference between the width in the flexible/semi-flexible blend and the width in the symmetric flexible mixture increases in accord with the Helfand Sapse description. This is confirmed by SCF calculations (c.f. Fig. 8), where we have assumed that the effective coordination numbers are temperature independent. However, upon increasing the incompatibility further $(\epsilon>0.082)$, one finds that an increase of the statistical segment length results in a smaller interfacial width of the asymmetric blend in qualitative contrast to the predictions of the Gaussian model.

\subsection{Distribution of chain ends and orientations}

The enrichment of chain ends at the center of the interface 30 and at hard walls 46, 47, 48, 49 has attracted abiding interest. Chain ends are important for the interdiffusion and healing properties at interfaces between long polymers [6]. They also play an important role for reactions at interfaces. In many experimental systems, chain ends have slightly different interactions than inner chain segments, which might result in a modification of the interfacial properties. On the theoretical side, the behavior of chain ends is related to corrections to the ground state approximation. Therefore it is a sensitive test for a quantitative theoretical description. Chain end effects give rise to large corrections to the interfacial width and tension, and they also play an important role for long range interactions between interfaces 43. The distribution of chain ends for symmetric blends has been investigated by Monte Carlo simulations 30, and in the framework of SCFT for Gaussian chains $[6$, 17. In Fig. 9 the simulational results and the SCF calculations are presented; both agree almost quantitatively. As in symmetric blends, chain ends are enriched at the center of the interface, and this effect goes along with a depletion away from the interface. The fact that the depletion zone in the wings shifts outwards with increasing chain length, indicates that the length scale of the rearrangement of chain ends is the radius of gyration. A-polymers stick their ends into the B-rich phase and vice versa. The effect on the semi-flexible chains becomes more pronounced with growing stiffness, while the A-polymers are hardly influenced by the stiffness of the B-polymers.

The instantaneous shape of a polymer coil is a prolate ellipsoid [30]. Polymers orient themselves by putting their ends preferentially at the center of the interface. This is quantified by the orientational parameter 30 for the end-to-end 
vector (cf. Fig. 10):

$$
q_{e}(z)=\frac{3\left\langle R_{z}^{2}\right\rangle_{z}-\left\langle\vec{R}^{2}\right\rangle_{z}}{2\left\langle\vec{R}^{2}\right\rangle_{z}}
$$

where the outer index $z$ at the brackets denotes the $\mathrm{z}$ coordinate of the midpoint of the end-to-end vector $\vec{R}$, and the inner indices its Cartesian components. The chains align their two long axis parallel to the interface in their majority phases, similar to the behavior at a hard wall. The chain orientation of semi-flexible polymers increases for growing stiffness, while the flexible A-polymers are not affected. The agreement between Monte Carlo simulation and SCF calculations is again almost quantitative. In the SCF framework, the orientations of the chains in the minority phase is accessible. The polymers align perpendicular to the interface, as to reach with one end their corresponding bulk phase. The length scale of the ordering increases with the bending energy $f$ and with chain length $N$.

The orientation of individual bond vectors $q_{b}$ shows a similar behavior. Bonds align parallel to the interface; the effect for the semi-flexible component grows with increasing bending energy and its range is largely independent of the chain length. The agreement between simulations and SCF calculations is very good. The Gaussian chain model cannot predict any nonzero orientation of the bonds. The orientation of bonds in our model is, in fact, much smaller than for the end-to-end distance [30].

In contrast to the width of the density profile, the spatial range over which the orientation of bonds extends grows upon increasing the bending energy. Therefore, the orientational width and the width of the composition profile are two independent microscopic length scales.

\section{Conclusions and outlook}

In summary, we have presented extensive simulations of highly incompatible polymers with different stiffness. The local structure of the interface has been characterized by density profiles of different monomer species and chain ends and orientational profiles of whole chains and individual bonds. The interfacial tension has been measured via analyzing the spectrum of capillary fluctuations. Using the pair correlation functions of the pure components and a random-packing like assumption for the intermolecular contacts between different species, we have extracted an effective Flory-Huggins parameter, which takes account of the stiffness dependence of the structure of the polymeric fluid. The effective Flory-Huggins parameter grows upon increasing the stiffness, because back folding is less probable and the number of intermolecular contacts increases respectively.

This effective Flory Huggins parameter was then employed in SCF calculations, as well as the chain conformations in the pure melt. These calculation 
incorporate the chain structure on all length scales via a partial enumeration scheme; there is no free parameter in describing the chain architecture. Using the detailed local structure of the bulk (as obtained by simulations) in the SCF calculations, we predict the interfacial properties.

Monte Carlo results and SCF calculations for the interfacial tension, the excess interfacial energy, the redistribution of chain ends and orientations of whole chains and individual bonds agree very well provided that the analysis accounts for capillary fluctuations. However, comparing our results to the analytical predictions of the Gaussian chain model for infinite chain length, we find qualitative deviations, especially for the dependence of the interfacial width on the chain stiffness. This finding might be important for extracting the Flory-Huggins parameter from interfacial profiles in highly incompatible polymer blends. Therefore, our results emphasize that the local structure, both of the underlying monomer fluid and of the chain architecture, is important for a quantitative description.

The radius of gyration determines the range of orientation of whole chains and the distribution of chain ends. Furthermore, we identify two independent microscopic length scales of the interfacial profile; one controls the width of the monomer density profile, the other corresponds to the range of orientations. This behavior resembles the findings in symmetric blends of worm-like chains in the limit $\kappa \chi \gg 1[16$ and the behavior of a homopolymer melt at a hard wall which is the limiting case for infinite incompatibility. However, in the present study this behavior is found for a different model which can be described neither by Gaussian nor by worm-like statistics on small length scales. Deviations from the Gaussian model occur under rather mild conditions which correspond roughly to $\kappa \chi=0.18 \cdots 0.47$ in the equivalent worm-like chain model. Furthermore our self consistent field approach as well as the simulation techniques are applicable to arbitrary chain architecture 19.

Assuming that the chain conformations and the local fluid structure are approximately independent of temperature, we have extended the self consistent field calculations to other incompatibilities. The results indicate that chain architecture becomes important when its length scale is comparable with the interfacial width. At very high incompatibility, increasing the stiffness of the semi-flexible component results in a decrease of the interfacial width. How-

ever, the Gaussian chain results and our calculations, which take account of the explicit chain architecture on all length scales, agree better for lower incompatibilities, where the interfacial width is much larger than the persistence length.

\section{Acknowledgment}

It is a great pleasure to thank K. Binder, G.S. Grest and F. Schmid for helpful and stimulating discussion, and M. Schick for critical reading of the manuscript. Generous access to the CRAY T3E at the San Diego Supercomputer Cen- 
ter (through a grant to M. Schick) is also gratefully acknowledged. M.M. thanks the Bundesministerium für Forschung, Technologie, Bildung und Wissenschaft(BMBF) for support under grant No. 03N8008C. A.W. thanks the Deutsche Forschungsgemeinschaft for support under grant number Bi 314/3.

\section{References}

[1] D.S. Walsh, J.S. Higgins, A. Maconnachie (eds.) Polymer Blends and Mixtures, Martinus Nijhoff, Dordrecht (1985). L.A. Kleintjens and P.J. Lemstra (eds.) Integration of Fundamental Polymer Science and Technology, Elsevier, Amsterdam (1986).

[2] M. Stamm and D.W. Schubert, Ann. Rev. Mat. Sci 25, 326 (1995). D.W. Schubert, V. Abetz, M. Stamm , T. Hack, W. Siol, Macromolecules 28, 2519 (1995).

[3] B. Löwenhaupt and G.P. Hellmann, Colloid \& Polymer Sci. 268, 885 (1990).

[4] H.E. Hermes, J.S. Higgins and D.G. Bucknall, Polymer 38, 985 (1997).

[5] K.H. Dai and E.J. Kramer, Polymer 35, 157 (1994). ibid Macromolecules 25, 220 (1992).

[6] D.T. Wu, G.H. Fredrickson, and J.P. Carton, J.Chem.Phys 104, 6387 (1996). D.T. Wu, G.H. Fredrickson, J.P. Carton, A. Ajdari, and L. Leibler, J.Poly.Sci 33B, 2373 (1996).

[7] F.S. Bates and G.H. Fredrickson, Macromolecules 27, 1065 (1994). F.S. Bates, M.F. Schulz, and J.H. Rosedale, Macromolecules 25, 5547 (1992).

[8] W.W. Graessley, R. Krishnamoorti, G.C. Reichart, N.P.Balsara, L.J. Fetters, and D.J. Lohse, Macromolecules 28, 1260 (1995) R. Krishnamoorti, W.W. Graessley, L.J. Fetters, R.G. Garner, and D.J. Lohse, Macromolecules 28, 1252 (1995).

[9] G.H. Fredrickson, A.J. Liu, and F.S. Bates, Macromolecules 27, 2503 (1994). G.H. Fredrickson and A.J. Liu, J.Polym.Sci. B33, 1203 (1995).

[10] C. Singh and K.S. Schweizer, J.Chem.Phys. 103, 5814 (1995). C. Singh and K.S. Schweizer, Macromolecules 30, 1490 (1997). J.D. Weinhold, S.K. Kumar, C. Singh and K.S. Schweizer, J.Chem.Phys. 103, 9460 (1995).

[11] K.F. Freed and J. Dudowicz, Macromolecules 29, 625 (1996).

[12] M. Müller, Macromolecules 28, 6556 (1995).

[13] E. Helfand and A.M. Sapse, J.Chem.Phys. 62, 1329 (1975). 
[14] A.I. Pesci and K.F. Freed, J.Chem.Phys. 90, 2017 (1989).

[15] N. Saito, K. Takahashi, and Y. Yunoki, J.Phys.Soc.Jpn. 22, 219 (1967).

[16] D.C. Morse and G.H. Fredrickson, Phys.Rev.Lett. 73, 3235 (1994).

[17] F. Schmid and M. Müller, Macromolecules 28, 8639 (1995).

[18] I. Szleifer, A. Ben-Shaul, and W.M. Gelbhart, J.Chem.Phys. 85, 5345 (1986), ibid. 86, 7094 (1987).

[19] M. Müller and M. Schick, Macromolecules 29, 8900 (1996).

[20] M. Müller and M. Schick, in preparation.

[21] I. Carmesin, K. Kremer, Macromolecules 21, 2819 (1988). H.P. Deutsch and K. Binder, J.Chem.Phys. 94, 2294 (1991).

[22] P.J. Flory, Statistical Mechanics of Chain Molecules, Interscience Publishers, N.Y. (1969).

[23] M. Müller and K. Binder, Macromolecules 28, 1825 (1995).

[24] The correlation hole effect also results in a reduction of the effective coordination number with increasing chain length 23.

[25] A. Yethiraj and K.S. Schweizer, J.Chem.Phys. 97, 5927 (1992). K.S. Schweizer and A. Yethiraj, J.Chem.Phys. 98, 9053 and 9080 (1993).

[26] A.N. Semenov, Macromolecules 26, 6617 (1993). ibid. 27, 2732 (1994).

[27] W. Helfrich, Z.Naturforsch. 28c, 693 (1973). P.B. Canham, J.Teor.Biol. 26, 61 (1970). E. Evans, Biophys.J. 14, 923 (1974).

[28] A. Werner, F. Schmid, M. Müller, and K. Binder, in preparation.

[29] The reduction of the measured $\mathrm{AB}$ coordination number upon increasing the lateral system size can be rationalized as follows. Assume, that the intrinsic coordination number $z_{\text {intr }}$ has no spatial dependence, the measured value has the form:

$$
\langle z\rangle=z_{\mathrm{intr}} \frac{\left\langle\phi_{A} \phi_{B}\right\rangle}{\left\langle\phi_{A}\right\rangle\left\langle\phi_{B}\right\rangle}
$$

The average in the simulation corresponds to a folding with the distribution of the deviation of local interfacial position from its lateral mean 26. The effect of this folding becomes the smaller the smaller the lateral system size is. Right at the center of the interface $\phi_{A}$ and $\phi_{B}$ have almost no curvature, whereas the product $\phi_{A} \phi_{B}$ has a large negative curvature (a maximum). Therefore $\left\langle\phi_{A} \phi_{B}\right\rangle\left\langle\left\langle\phi_{A}\right\rangle\left\langle\phi_{B}\right\rangle\right.$, i.e. the measured value is smaller than the intrinsic one. 
[30] M. Müller, K. Binder, and W. Oed, Faraday Trans. 91, 2369 (1995).

[31] G.S. Grest, M.D. Lacasse, K. Kremer, and A.M. Gupta, J.Chem.Phys. 105, 10583 (1996). G.S. Grest, M.D. Lacasse, and M. Murat, MRS Bulletin 22, 27 (1997). R.B. Pandey, A. Milchev, and K. Binder, Macromolecules 29,1194 (1997).

[32] G.J.A. Ypma, P. Cifra, E. Nies, and A.R.D. van Bergen, Macromolecules 29, 1252 (1996). P. Cifra, E. Nies, and F.E. Karasz, Macromolecules 27, 1166 (1994).

[33] M. Müller and M. Schick, J.Chem.Phys. 105, 8885 (1996), ibid. 105, 8282 (1996).

[34] E. Helfand and Y. Tagami, J.Polym.Sci.,Polym.Lett. 9, 741 (1971). E. Helfand and Y. Tagami, J.Chem.Phys. 56, 3592 (1972).

[35] J. Noolandi and K.M. Hong, Macromolecules 14, 727 (1981), ibid 15, 483 (1982).

[36] K.R. Shull, Macromolecules 26, 2346 (1993).

[37] E. Helfand, J.Chem.Phys 62, 999 (1975).

[38] The compressibility of the athermal system has been measured in M. Müller and W. Paul, J.Chem.Phys. 100, 719 (1994). $\zeta=-\Phi \frac{\partial^{2} s}{\partial \Phi^{2}}$, where $s$ denotes the entropy density.

[39] S.F. Edwards, Proc.Phys.Soc. 85, 613 (1965).

[40] M.W. Matsen and M. Schick, Phys.Rev.Lett. 74, 4225 (1995).

[41] L. Leibler, Macromol.Chem.Macromol.Symp. 16, 1 (1988). S. Wu, Polym.Eng.Sci. 27, 335 (1987).

[42] S.T. Milner, MRS Bulletin 22, 38 (1997).

[43] A.V. Ermoshkin and A.N Semenov, Macromolecules 29, 6294 (1996). A.N. Semenov, J.Phys.II 6, 1759 (1997).

[44] C. Creton, E.J. Kramer, and G. Hadziioannou, Macromolecules 24, 1846 (1991). J. Washiyama, C. Creton, and E.J. Kramer, Macromolecules 25, 4751 (1992).

[45] Upon increasing the stiffness disparity, the profiles of our compressible blend become slightly asymmetric. Fitting a tanh-profile to the A- and B-monomer density, one finds that the B-profile is about $1 \%$ wider $(f=2)$ than the A-profile. This effect is observed in simulations and SCF calculations. 
[46] C. Jalbert, J. Koberstein, A. Hariharan and S.K. Kumar, Macromolecules 30, 4481 (1997).

[47] I. Bitsanis, G. Hadziioannou, J.Chem.Phys. 92, 3827 (1990).

[48] F. Schmid, J.Chem.Phys. 104, 9191 (1996).

[49] J. Baschnagel and K. Binder, Macromolecules 28, 6808 (1995).

\begin{tabular}{|c|c|c|c|c|c|c|c|c|c|c|c|}
\hline$N$ & $f$ & $\left\langle b^{2}\right\rangle$ & $R^{2}$ & $R_{g}^{2}$ & $z_{B B}$ & $\langle e\rangle / k_{B} T$ & $e_{s} / k_{B} T$ & $w_{a}$ & $w_{r}$ & $w_{e}$ & $w_{\text {scf }}$ \\
\hline \hline 32 & 0.0 & 6.92 & 290.4 & 48.8 & 2.65 & -0.00732 & 0.0290 & 4.77 & 3.88 & 3.51 & 3.11 \\
& 0.5 & 6.88 & 350.1 & 58.1 & 2.84 & -0.00731 & 0.0293 & 4.63 & 3.83 & 3.40 & 3.13 \\
& 1.0 & 6.86 & 431.8 & 70.8 & 3.02 & -0.00730 & 0.0300 & 4.57 & 3.80 & 3.38 & 3.16 \\
& 1.5 & 6.84 & 536.9 & 86.5 & 3.17 & -0.00730 & 0.0304 & 4.49 & 3.77 & 3.34 & 3.19 \\
& 2.0 & 6.84 & 665.2 & 105.1 & 3.29 & -0.00730 & 0.0310 & 4.53 & 3.75 & 3.34 & 3.23 \\
\hline 64 & 0.0 & 6.92 & 609.3 & 101.7 & 2.53 & -0.00732 & & & & & \\
& 1.0 & 6.86 & 987.3 & 148.3 & 2.92 & -0.00730 & 0.0265 & 4.13 & 3.43 & 3.11 & 2.99
\end{tabular}

Table 1: Single chain conformations and interfacial properties as a function of the bending energy $f$. Interfacial data refer to blends of flexible $(f=0)$ and semi-flexible ( $f$ as indicated) chains. $\left\langle b^{2}\right\rangle$ : mean squared bond length, $R^{2}$ : mean squared end-to-end distance, $R_{g}^{2}$ mean squared radius of gyration, $z_{B B}$ effective intermolecular coordination number as measured in simulations of the bulk system, $\langle e\rangle / k_{B} T$ : bulk energy density, $e_{s} / k_{B} T$ : interfacial energy excess per unit area, $w_{a}$ apparent interfacial with for $L=64, w_{r}$ reduced interfacial width for block size $B=16, w_{e}$ estimated interfacial with from the excess energy, $w_{\text {scf }}$ interfacial width in the SCF calculations. 


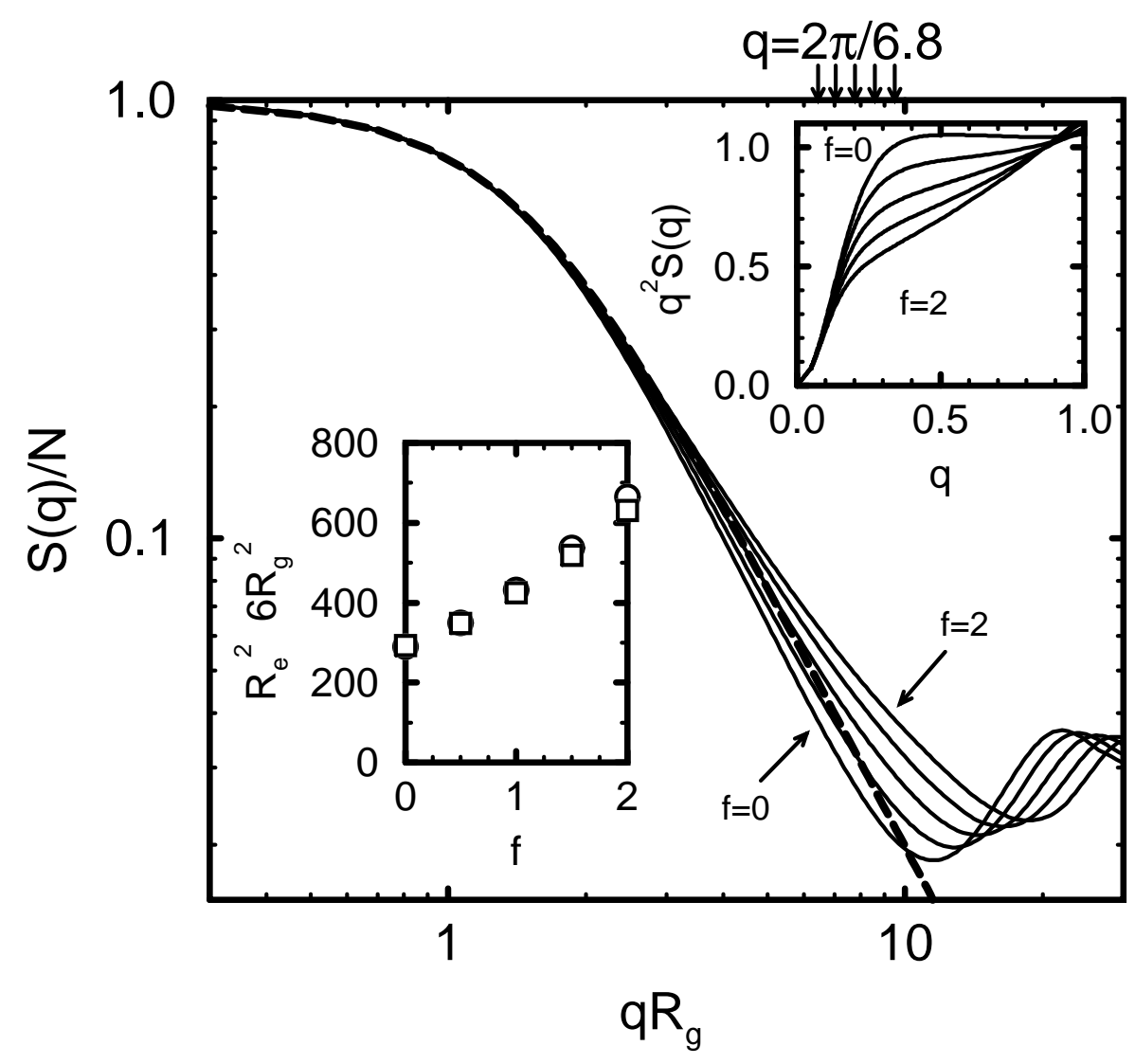

Figure 1: Single chain structure function $S(q)$ for bending energies $f=0.0,0.5,1.0,1.5$, and 2.0 (solid lines). The dashed line represents the Debye function. The arrows, on top of the figure, characterize the interfacial length scale $\left(2 w_{\mathrm{SCF}}=6.8\right)$.

Left inset: Mean squared end-to-end distance (circles) and radius of gyration (squares) as a function of the bending energy $f$. Upon increasing $f$, the chain extension grows about a factor 1.5. Note that the ratio $R_{e}^{2} / R_{g}^{2}$ remains close to its Gaussian value 6 .

Right inset: Kratky plot of the single chain structure factor $S(q)$ for $f=0.0,0.5,1.0,1.5$, and 2.0 from top to bottom. A plateau, characteristic of Gaussian statistics, is only found for $f=0$ (flexible chains), whereas there are pronounced deviations for semi-flexible chains. 


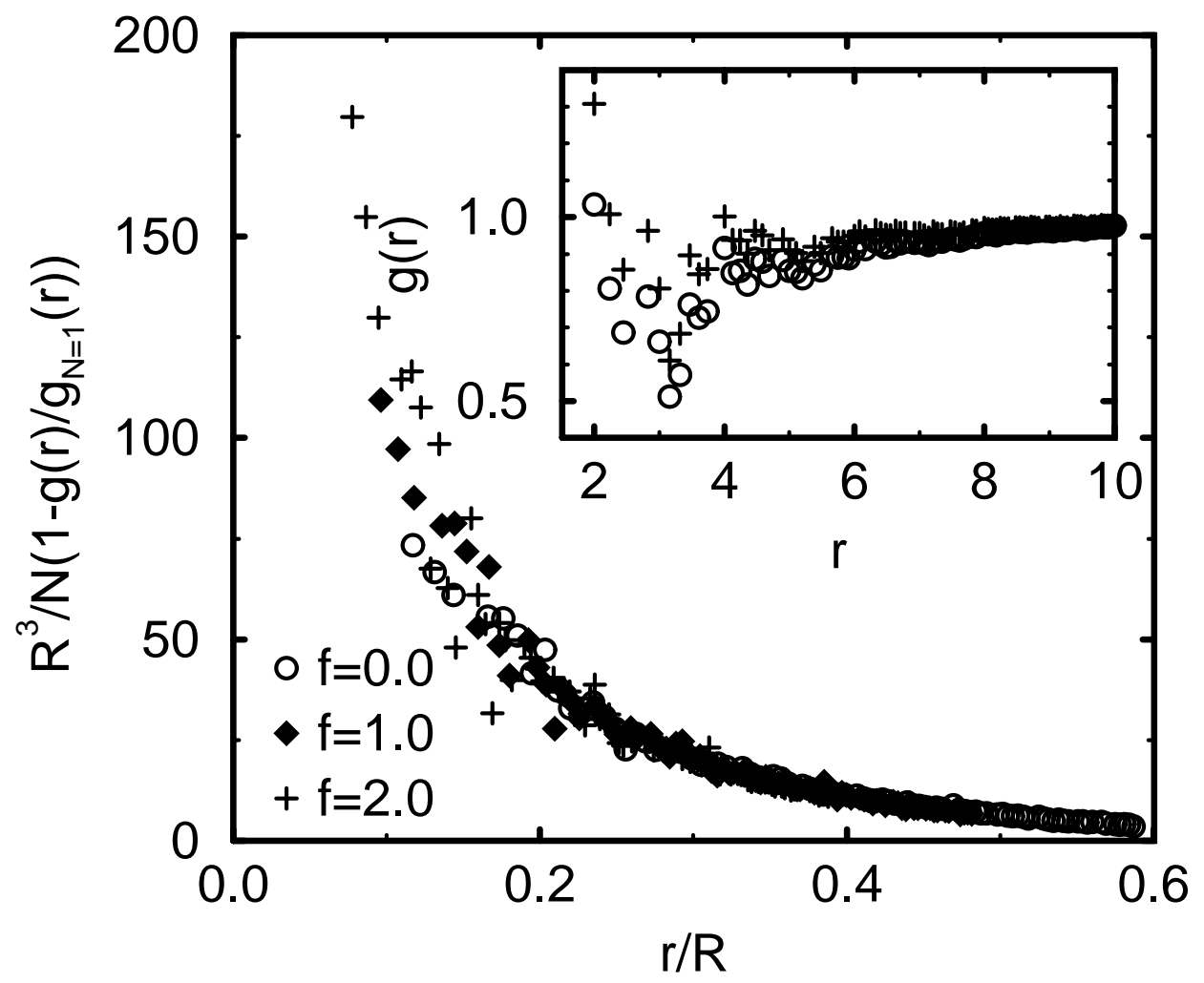

Figure 2: Scaling plot for the intermolecular pair-correlation function for bending energies $f=0,1$ and 2. The inset shows the pair-correlation function for the lowest and the highest bending energy studied. 


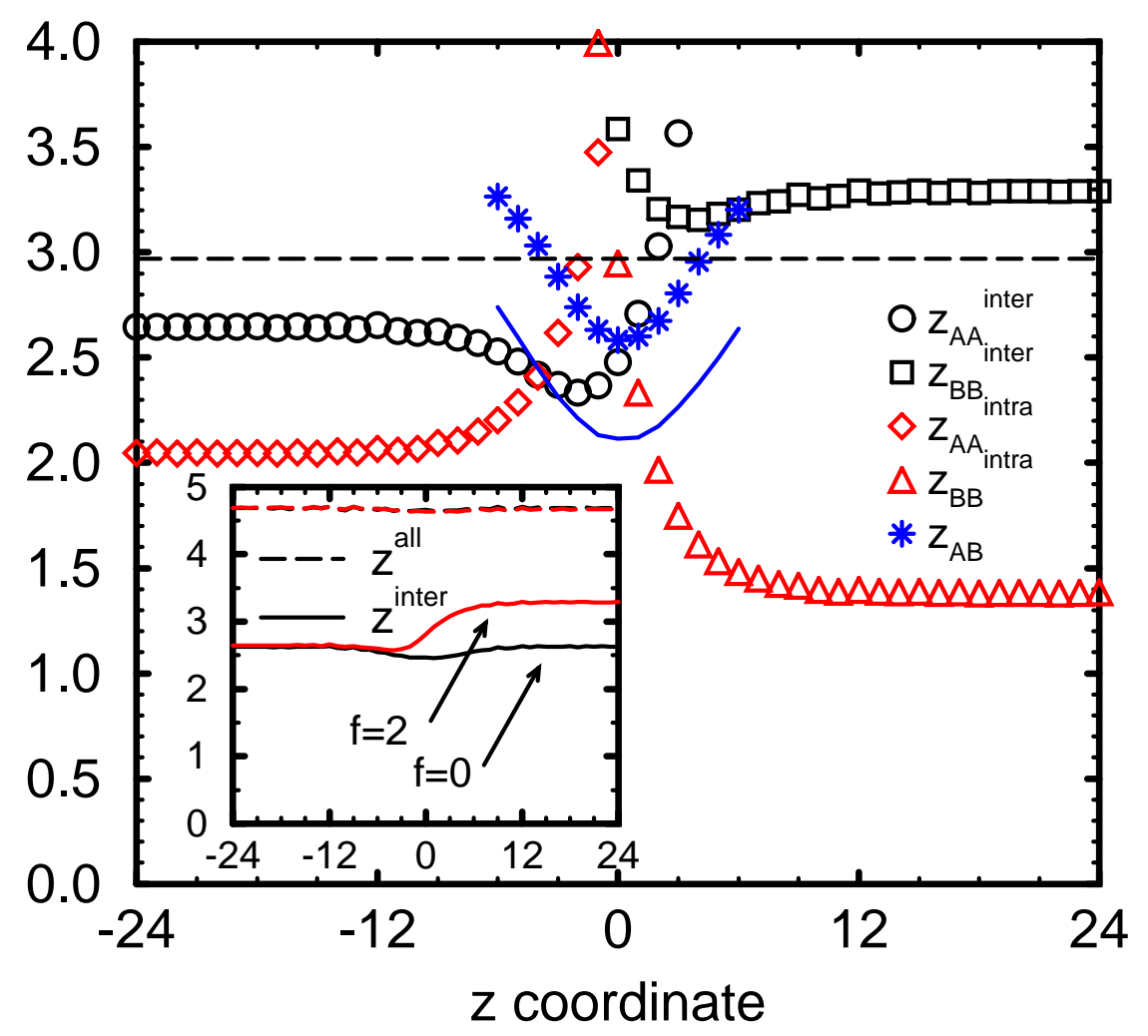

Figure 3: Reduced profiles of the coordination numbers of inter- and intrachain contacts for the bending energy $f=2$ The flexible component is on the left side of the interface. The line presents the apparent profile for the $\mathrm{AB}$ intermolecular contacts. The dashed line is the random mixing like approximation for the $\mathrm{AB}$ intermolecular contacts. The inset shows the total number of contacts and number of interchain contacts. 


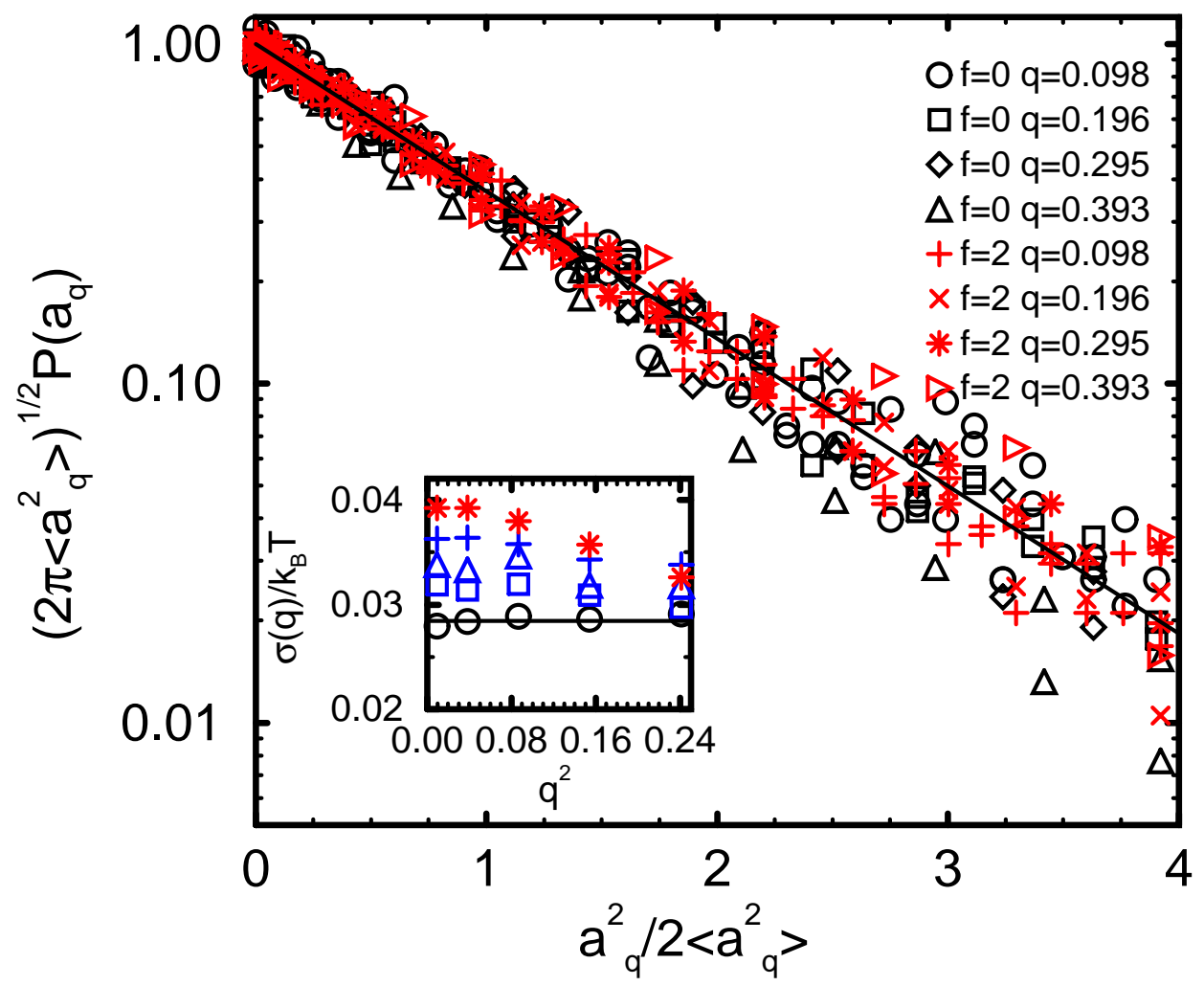

Figure 4: Probability distribution of Fourier components of the local interfacial profile for the 4 smallest wave-vectors $q$ and bending energies $f=0$ and 2 , using the $1 / q^{2}$ dependence of the variance. The solid line represents the expected Gaussian distribution.

inset: Inverse width of the distribution $\sigma(q)=\frac{2}{L^{2} q^{2}\left\langle a_{q}^{2}\right\rangle}$ for all bending energies (circles $f=0.0$, squares $f=0.5$, triangles $f=1.0$, crosses $f=1.5$, and stars $f=2.0$ ) and the smallest $q$ values. The 3 smallest wave-vectors were used to determine the interfacial tension $\sigma$. The Helfrich Hamiltonian describes the data down to smaller $q$ values for the symmetric blend than for $f=2$. The solid line denotes the independent estimate for the symmetric blend $(f=0)$ from simulations in the semi-grandcanonical ensemble. 


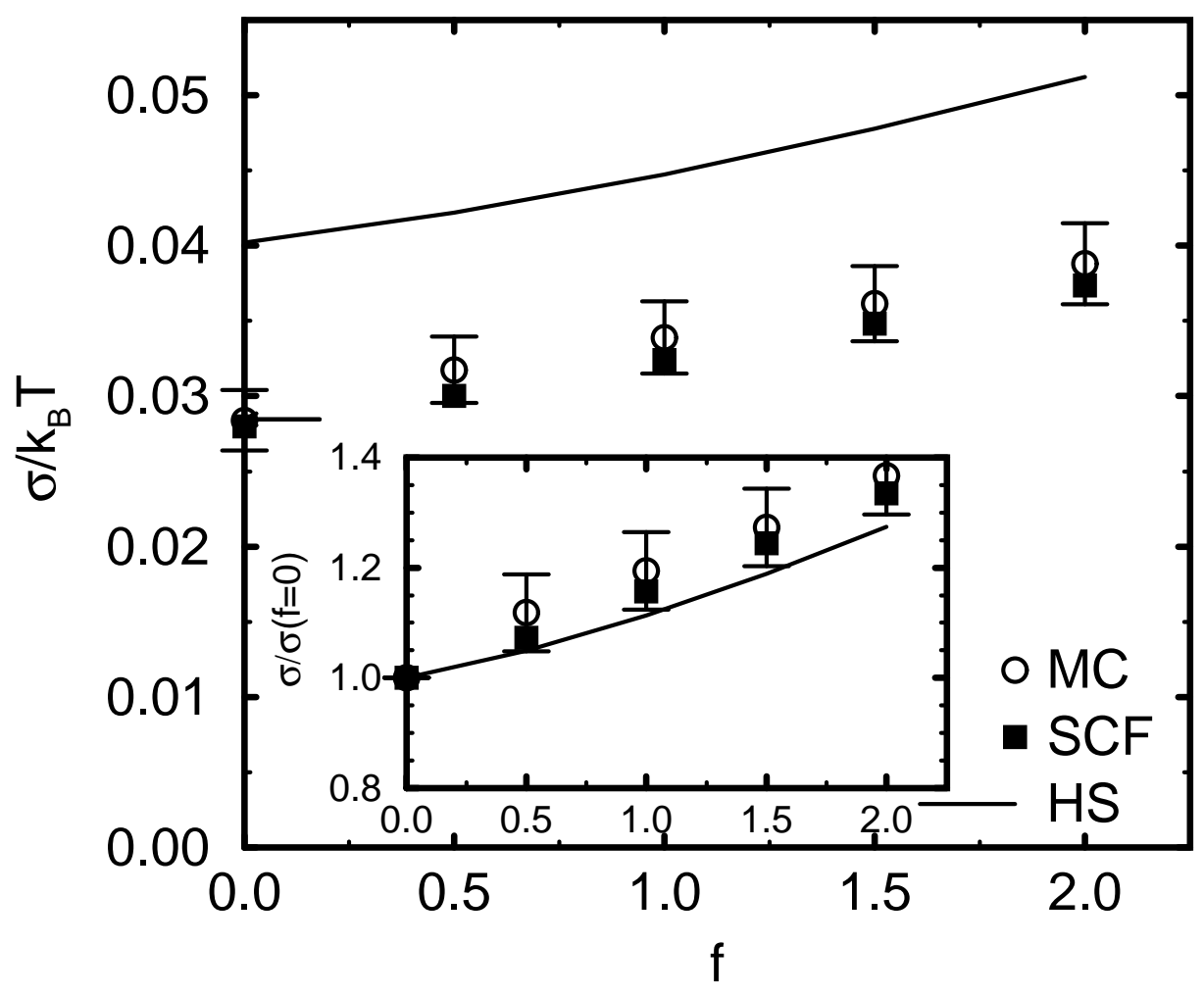

Figure 5: Interfacial tensions $\sigma$ for all bending energies. Circles represent Monte Carlo estimates obtained from the capillary fluctuation spectrum, squares denote the values of the self consistent field calculations, and solid lines show the Helfand-Sapse prediction (where we have neglected the architecture dependence to the Flory-Huggins parameter i.e. $\chi=0.265$ for all $f$ ). 


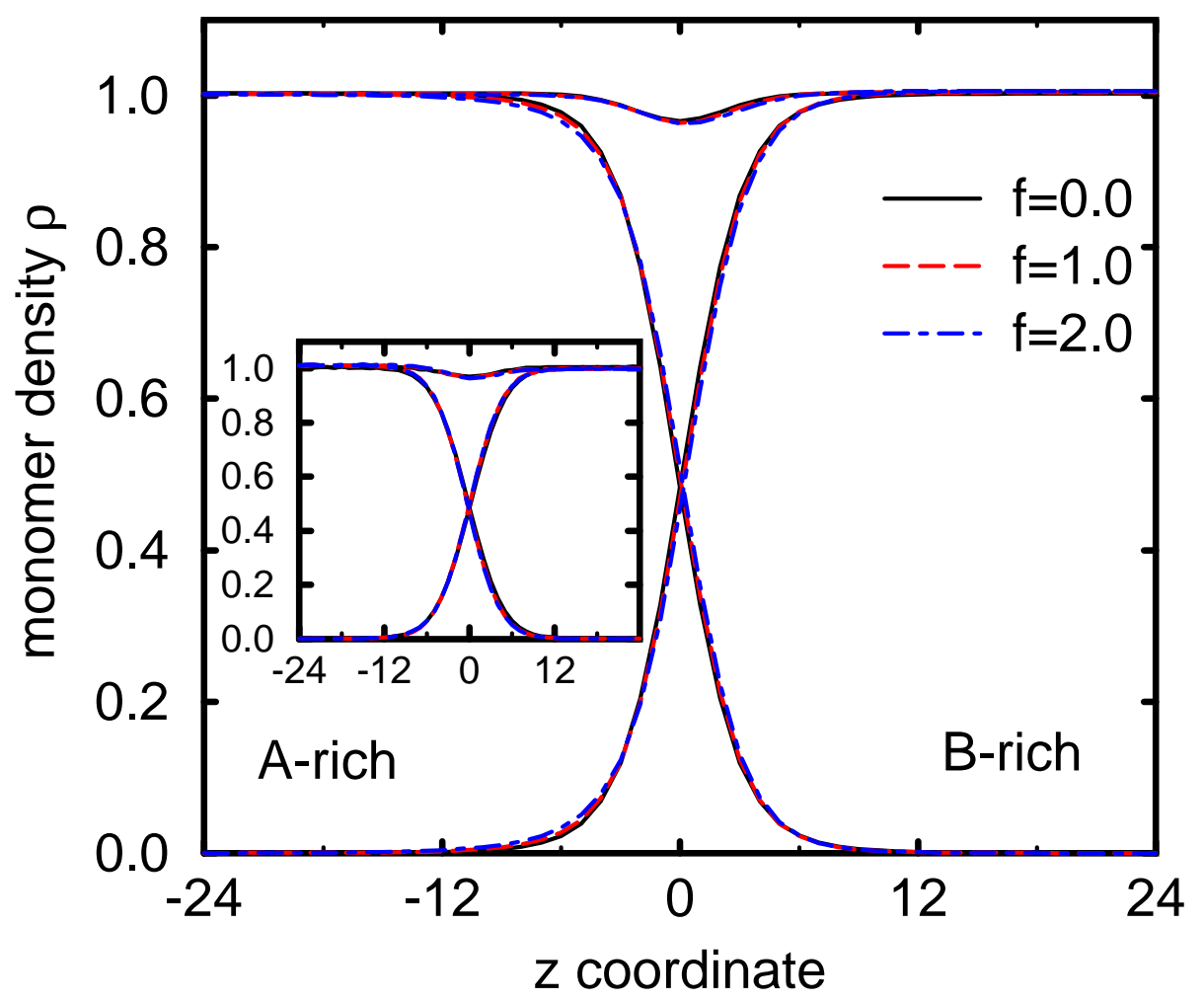

Figure 6: A- and B-monomer density, and total density profiles for bending energies $f=0,1$, and 2 from SCF calculations for chain length $N=32$. The apparent profiles in the Monte Carlo simulations are shown in the inset). The Monte Carlo profiles are broader than the self consistent field results, but in both cases there is almost no dependence on the bending energy $f$. 


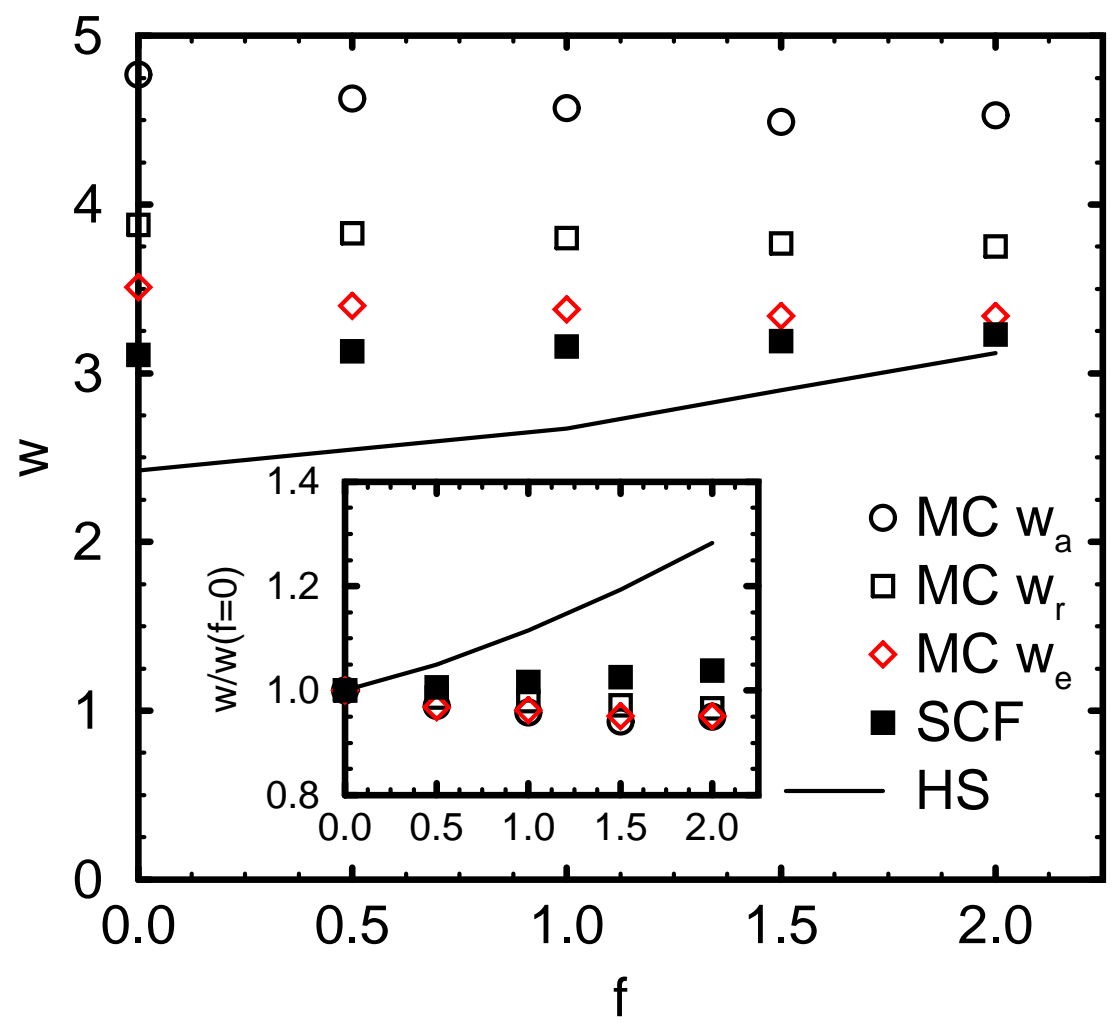

Figure 7: Interfacial width as a function of bending energy $f$ for chain length $N=32$. Circles denote the apparent width $w_{a}$ in the Monte Carlo simulations $(L=64)$, squares represent the Monte Carlo result $w_{r}$ from the reduced profiles $(B=16)$, diamonds show the estimate from the interfacial excess energy, filled squares mark the results of the self consistent field calculations, and the solid line shows the Helfand-Sapse prediction $(\chi=0.265$ for all $f$ ). The inset presents the same data normalized by the interfacial width of the symmetric blend. 


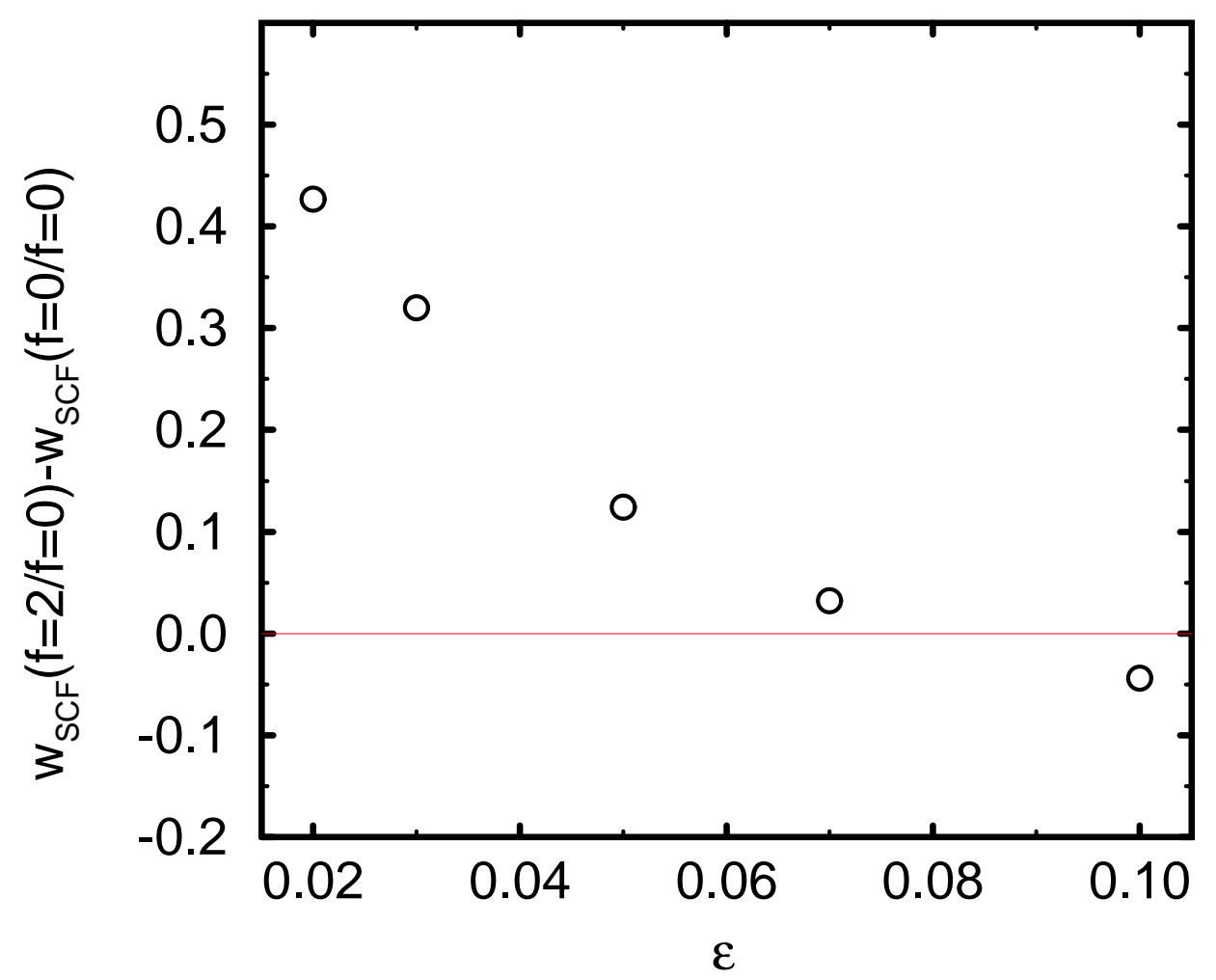

Figure 8: Difference between the width of the $f=0 / f=2$ blend and the symmetric $f=0$ mixture in the self consistent field calculations. For $\epsilon<0.082$ the interfacial width increases upon increasing the statistical segment length of the semi-flexible component, whereas at higher incompatibilities the width decreases. 

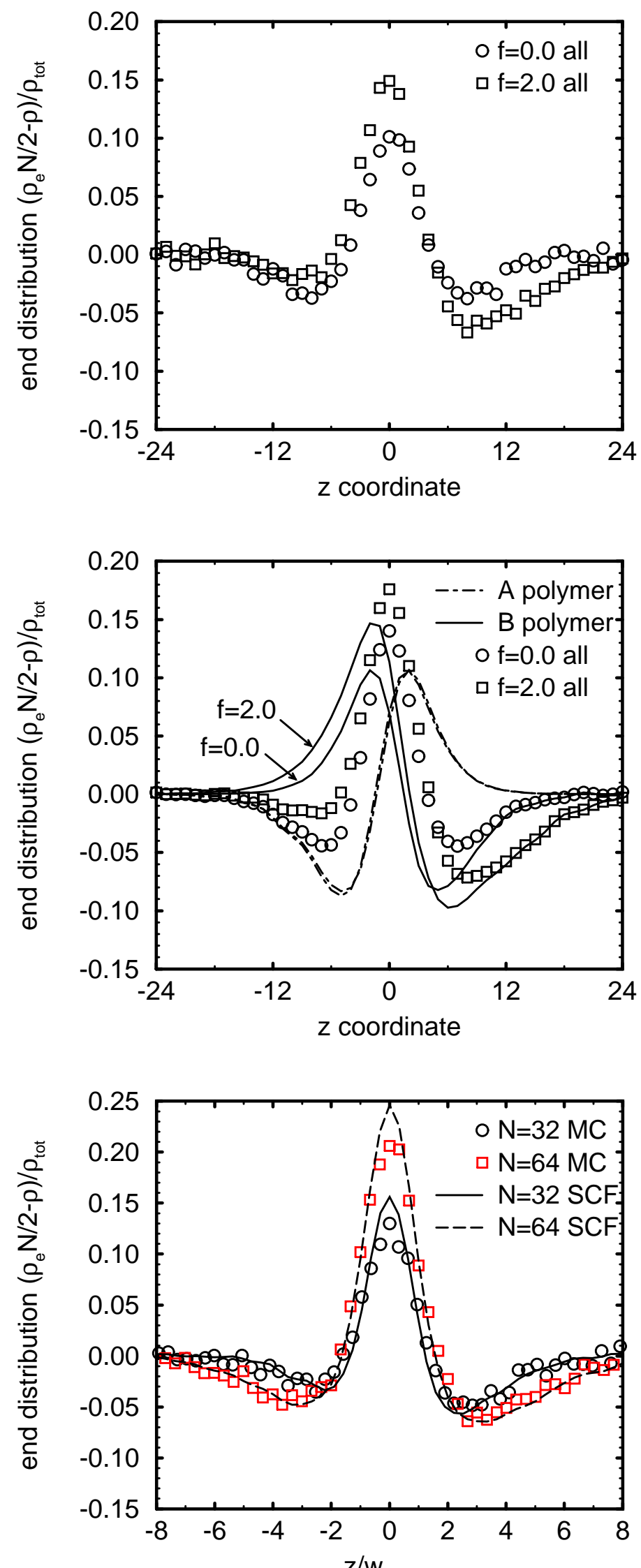

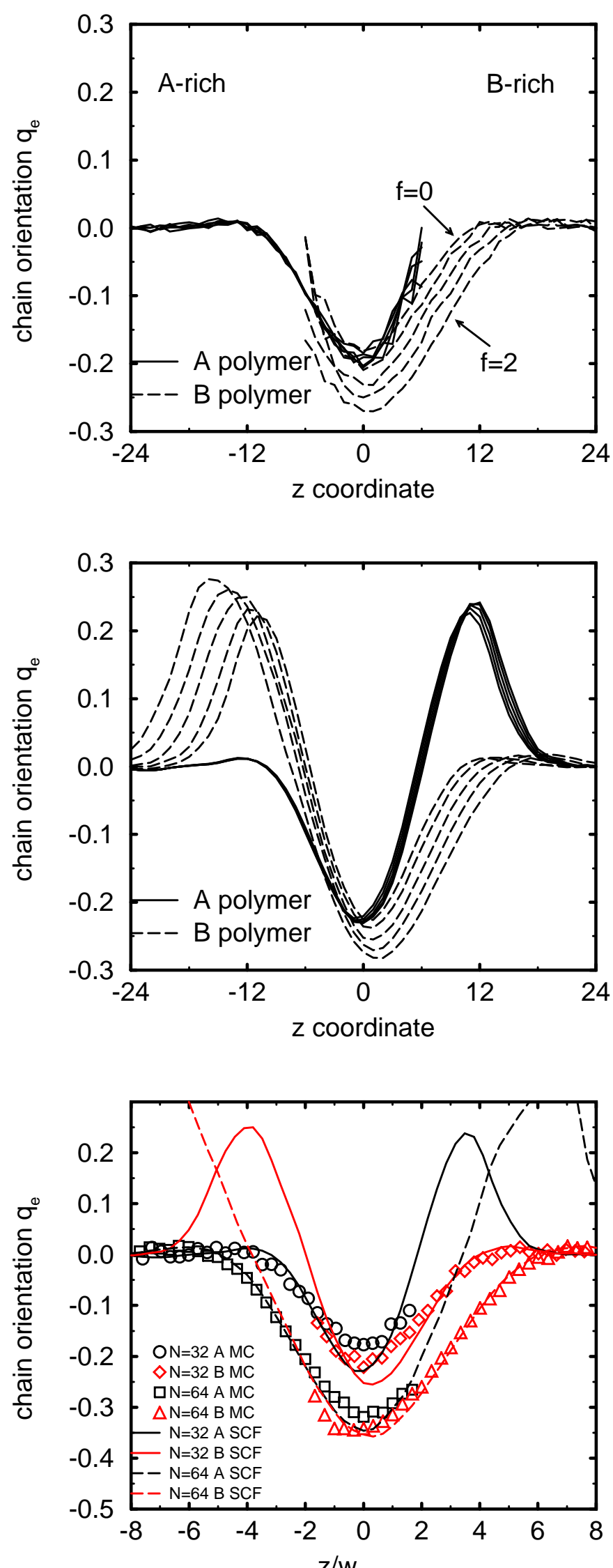

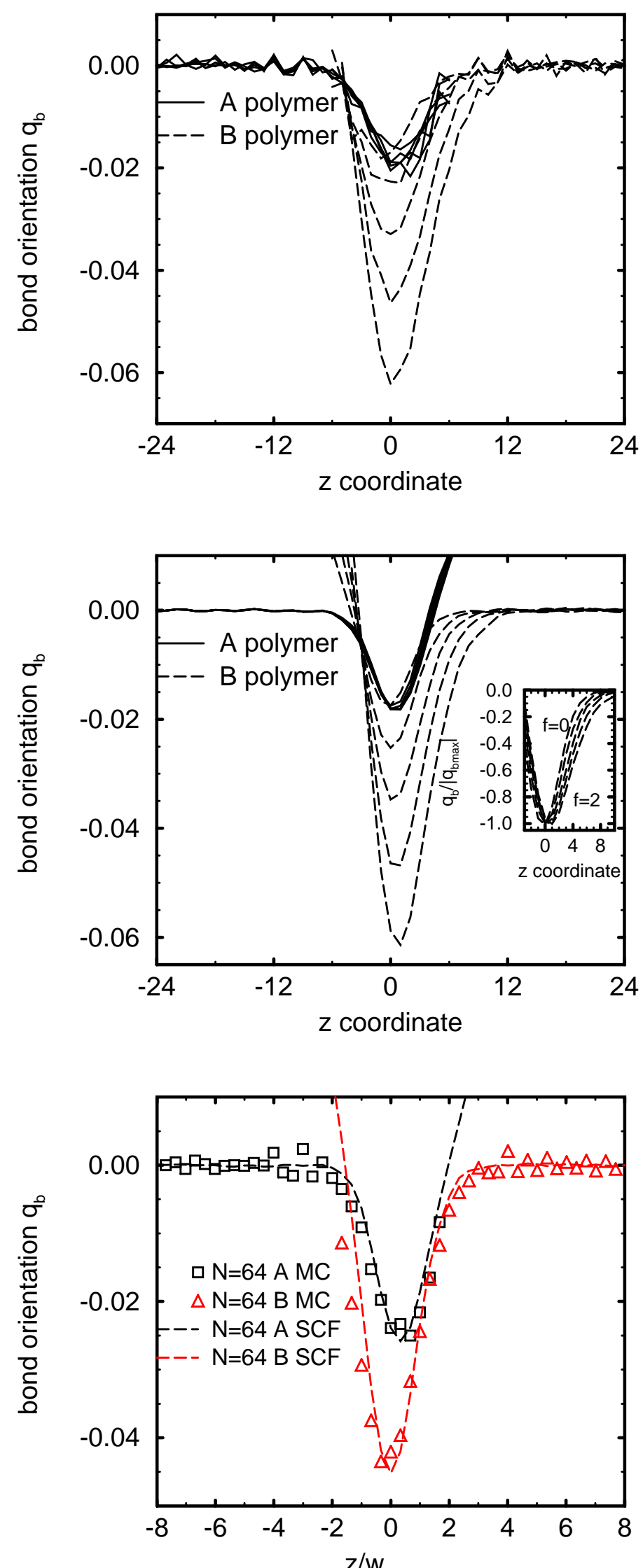